\title{
Numerical investigation of phase change materials thermal capacitor for pipe flow
}

\author{
Jundika Candra Kurnia ${ }^{1, *}$, and Agus Pulung Sasmito ${ }^{2}$ \\ ${ }^{1}$ Department of Mechanical Engineering, Universiti Teknologi PETRONAS, 32610 Bandar Seri \\ Iskandar, Perak Darul Ridzuan, Malaysia. \\ ${ }^{2}$ Department of Mining and Materials Engineering, McGill University, 3450 University, Montreal, \\ QC, H3A2A7 Canada
}

\begin{abstract}
This study addresses the performance of phase change material as thermal capacitor. A computational fluid dynamics (CFD) model is developed to take into account the conjugate heat transfer between water as the heat transfer fluid (HTF) and PCM as thermal capacitor. A pulsating inlet temperature with constant inlet velocity is prescribed to represent temperature variation. The performance of thermal capacitor is evaluated by closely monitoring outlet temperature and comparing it with inlet temperature to examine the reduction in temperature fluctuation. To intensify heat transfer between HTF and PCM, extended surfaces (fins) are installed on PCM side. The results indicate that PCM thermal capacitor can reduce temperature fluctuation by $\sim 1{ }^{\circ} \mathrm{C}$. This reduction can be improved further when extended surface is installed with $\sim 1.5{ }^{\circ} \mathrm{C}$ reduction in temperature fluctuation is achieved. Moreover, it is found that the maximum temperature is delayed at the outlet due to slow conjugate heat transfer between HTF and PCM. Inlet velocity is found to have considerable influence of the temperature fluctuation reduction: Slower inlet velocity results in a better temperature fluctuation reduction. This study is expected to serve as a guideline in designing PCM-based thermal capacitor.
\end{abstract}

\section{Introduction}

Due to its capability of storing large amount of energy at minimum temperature rise, phase change materials have been widely adopted as energy storage medium [1]. Numerous phase change materials have been investigated as thermal energy storage ranging from organic materials over inorganic materials to eutectic materials [1-4]. In tandem, a number of PCM thermal energy storage configuration have been proposed, e.g. pipe storage, cylindrical storage, shell and tube storage, rectangular storage and triplex tube storage [2,57].

In addition to energy storage, there is a growing interest to utilize phase change materials as thermal capacitor or smoothing devices. Nardin et al [8] investigated the performance of aluminium as phase change materials to smooth the off-gas temperatures

\footnotetext{
* Corresponding author: jundika.kurnia@utp.edu.my
} 
from the electric arc furnace process of steel industry for energy recovery purposes. They found out that the variation of the off gas temperature is significantly reduced. The concept was further developed for continuous charge electric arc furnace process in study by Dal Magro et al [9]. The findings highlight that reduction in off gas temperature fluctuation results in the increase of the whole recovery system efficiency. This in turn reduces size and investment cost of the recovery system and also eliminating the environmental issues due to dioxins generations. Novikov et al. [10] evaluate the performance of PCM powder to smooth temperature peak produced by the electronic component. It was found that PCM powder reduce the peak temperature. Among various PCM powder evaluated in their study, it was revealed that erythritol is the most promising. Verdier et al [11] experimentally investigated the PCM thermal energy storage to Hybrid Solar Gas Turbine (HSGT) systems for smoothing of temperature variation. In their study, the main goal is to keep the outlet air temperature from the collector above $650{ }^{\circ} \mathrm{C}$.

These studies highlight the potential of PCM as thermal capacitor or temperature smoothing devices. Nevertheless, most of them focus on external flow, where the heat transfers fluid flow across bank of cylindrical tube container of PCM. It is therefore of interest to evaluate the effectiveness of phase change materials (PCM) as thermal capacitor or temperature smoothing device in a simple internal flow such as pipe flow. This study is conducted to evaluate the performance of PCM in reducing temperature variation of fluid flowing through straight pipe. To emulate the variation of temperature, a sinusoidal temperature profile with constant fluid velocity is prescribed at the inlet. The outlet temperature is monitored to evaluate the reduction in temperature variation. To enhance conjugate heat transfer between HTF and PCM and thus better smoothing, fin is installed in PCM side. The effect of inlet velocity will be evaluated as well.

\section{Mathematical model}

A thermal capacitor comprises HTF channel and pool of PCM is subjected to inlet temperature fluctuation, as illustrated in Fig.1. The PCM considered in this study is paraffin wax (n-octadecane) which has melting temperature around $28^{\circ} \mathrm{C}$.

\subsection{Governing equations}

The mathematical model incorporates conjugate heat transfer between water as HTF and PCM as thermal capacitor. In HTF, fluid flow and convective heat transfer is taken into account. The conservation of mass, momentum and energy in HTF are given by [12].

$$
\begin{gathered}
\frac{\partial \rho_{f}}{\partial t}+\nabla \cdot\left(\rho_{f} \mathbf{u}\right)=0 \\
\frac{\partial\left(\rho_{f} \mathbf{u}\right)}{\partial t}+\nabla \cdot\left(\rho_{f} \mathbf{u u}\right)=-\nabla p+\nabla\left[\mu_{f}\left(\nabla \mathbf{u}+(\nabla \mathbf{u})^{T}\right)\right]+\rho_{f} \mathbf{g} \\
\frac{\partial\left(\rho_{f} c_{p, f} T\right)}{\partial t}+\nabla \cdot\left(\rho_{f} c_{p, f} \mathbf{u} T\right)=\nabla \cdot\left(k_{f} \nabla T\right)
\end{gathered}
$$

where $\rho_{f}, \mu_{f}$, and $c_{p, f}$ are the density, dynamic viscosity and specific heat of the fluid, respectively. Meanwhile, $p$ is the pressure, $\mathbf{u}$ is the fluid velocity and $T$ is the temperature.

The conservation of mass, momentum and energy in PCM are given by [12]

$$
\frac{\partial \rho_{p}}{\partial t}+\nabla \cdot\left(\rho_{p} \mathbf{u}\right)=0
$$




$$
\begin{gathered}
\frac{\partial\left(\rho_{p} \mathbf{u}\right)}{\partial t}+\nabla \cdot\left(\rho_{p} \mathbf{u} \mathbf{u}\right)=-\nabla p+\nabla\left[\mu_{p}\left(\nabla \mathbf{u}+(\nabla \mathbf{u})^{T}\right)\right]+\rho_{p} \mathbf{g}+\mathbf{s}_{\text {mот }} \\
\frac{\partial\left(\rho_{p} H_{p}\right)}{\partial t}+\nabla \cdot\left(\rho_{p} \mathbf{u} H_{p}\right)=\nabla \cdot\left(k_{p} \nabla T\right)
\end{gathered}
$$

where $\rho_{p}$ and $\mu_{f}$, are the density and dynamic viscosity of the PCM, respectively. $H_{p}$ ans $\mathbf{s}_{\text {mom }}$ are additional terms added to momentum and energy equations to take into account reduced porosity in the mushy zone and latent heat of PCM.

a)

)

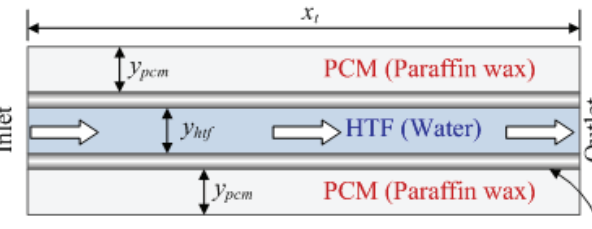

c)

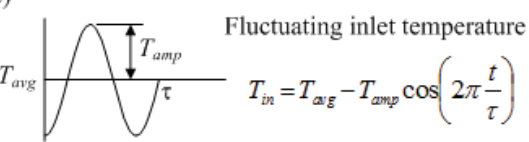

b)

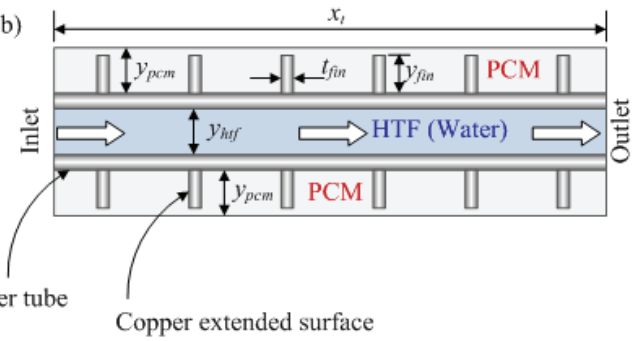

Fig. 1. Schematics of a) PCM thermal capacitor without fin, b) PCM thermal capacitor with fin and c) fluctuating inlet temperature.

\subsection{Constitutive relations}

Thermal properties of HTF (water) and PCM are strong function of temperature. Therefore a polynomial functions are adopted to represent this strong dependency ( please refer to our previous publications [13-15] for details polynomial function adopted in this study).

\subsection{Boundary conditions}

The boundary conditions imposed on the mathematical model are summarized as follows:

- Inlet: at the inlet we specify constant inlet velocity and fluctuating temperature.

- Outlet: at the outlet, we set pressure and stream-wise gradient of temperature to zero.

- HTF channel wall: no slip condition and coupled temperature is prescribed.

- PCM outer wall: no slip condition and zero heat flux is implemented.

\section{Numerical methodology}

The mathematical model is incorporated to the computational domain, created, meshed and labelled in ANSYS workbench and the solved by using finite volume solver, ANSYS Fluent 15. User-defined-function is added to the computational model to account for thermo physical properties of water and PCM. The model is solved with semi-implicit pressure linked equation (SIMPLE) algorithm, Presto discretization for pressure and second order upwind discretization for other parameter together with algebraic multi-grid method. The convergence criterion of $10^{-3}$ is set for continuity and momentum while the convergence of energy is set at $10^{-6}$. To ensure mesh independent result four different mesh amounting $2 \times$ $10^{4}, 4 \times 10^{4}, 6 \times 10^{4}$, and $8 \times 10^{4}$ are used and compared. The results indicate that no significant different is found between the result from $6 \times 10^{4}$ and $8 \times 10^{4}$ meshes. Hence mesh size of $6 \times 10^{4}$ is used for the remaining simulation. To assess the validity of the 
developed model, validation has been conducted for both PCM and HTF model, as has been presented in our previous work [13-16].

Table 1. Base case and operating parameters.

\begin{tabular}{llllll}
\hline Parameter & \multicolumn{1}{c}{ Value } & Units & Parameter & Value & \multicolumn{1}{c}{ Units } \\
\hline$T_{\text {avg }}$ & 300.65 & $\mathrm{~K}$ & $T_{m}$ & 300.65 & $\mathrm{~K}$ \\
$T_{\text {amp }}$ & 10 & $\mathrm{~K}$ & $p_{\text {out }}$ & 0 & $\mathrm{~Pa}$ (gage) \\
$U_{\text {in }}$ & $0.043,0.085,0.170$ & $\mathrm{~m} / \mathrm{s}$ & $y_{p c m}$ & 0.1 & $\mathrm{~m}$ \\
$L$ & 243,500 & $\mathrm{~J} / \mathrm{kg}$ & $y_{h t f}$ & 0.1 & $\mathrm{~m}$ \\
$c_{p, p}$ & 2160 & & $y_{\text {fin }}, t_{\text {fin }}$ & $0.08,0.02$ & $\mathrm{~m}$ \\
$k_{p}^{(s)}, k_{p}^{(l)}$ & $0.358,0.148$ & $\mathrm{~W} / \mathrm{m} \cdot \mathrm{K}$ & $t_{c u}$ & 0.02 & $\mathrm{~m}$ \\
$\zeta$ & 100,000 & - & $x_{t}$ & 0.5 & $\mathrm{~m}$ \\
\hline
\end{tabular}

\section{Results and discussion}

The computational simulations are carried out for steady flow with fluctuating inlet temperature. The operating conditions and important parameters are listed in Table 1. The inlet temperature fluctuates in sinusoidal manner with maximum temperature of 290.65 and minimum temperature of 310.65 with a period of 10 minutes.

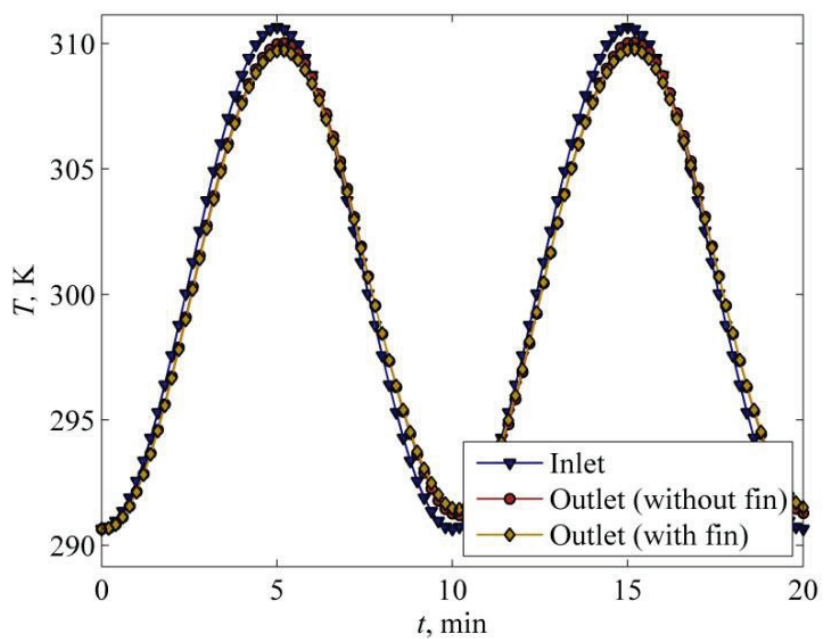

Fig. 2. Temperature fluctuation at inlet and outlet of PCM thermal capacitors at inlet Reynolds number of 1000 .

The performance of investigated thermal capacitor is presented in Fig. 2. As can be seen, the investigated thermal capacitor is capable in reducing the temperature fluctuation as mirrored by the lower maximum outlet temperature as compared to the inlet temperature. The reduction is, however, relatively marginal. For PCM thermal without fin, the maximum temperature is reduced by $\sim 1{ }^{\circ} \mathrm{C}$. When the fin is added to the PCM to improve the heat transfer, only marginal improvement is observed where the maximum temperature is reduced by $\sim 1.5^{\circ} \mathrm{C}$. Another interesting phenomenon that should be noted in Fig. 2 is the 
shifting of peak temperature: The maximum temperature at the outlet is delayed by $9 \mathrm{~s}$ and $7 \mathrm{~s}$ for PCM thermal capacitor without and with fin, respectively. This phenomenon is most likely attributed to the slow conjugate heat transfer between the heat transfer fluid and PCM. This is supported by the smaller delay for PCM thermal capacitor with fin as compared to that without fin.

a)
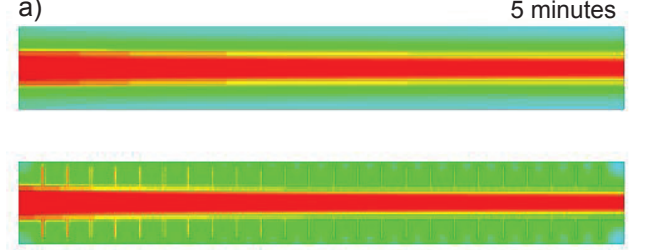

10 minutes
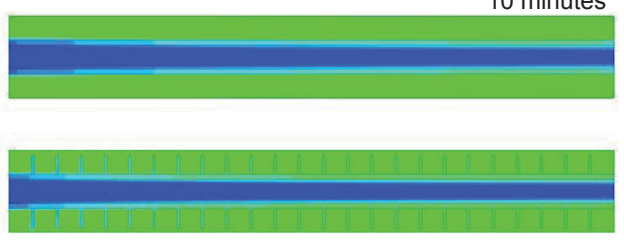

$\begin{array}{lllllllllll}290 & 292 & 294 & 296 & 298 & 300 & 302 & 304 & 306 & 308 & 310\end{array}$ b)
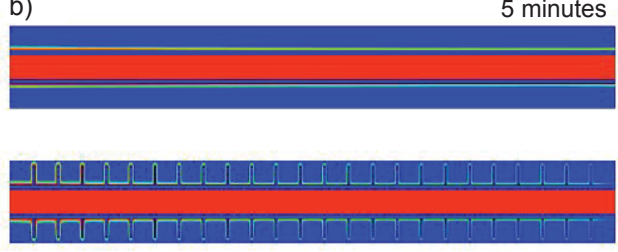

10 minutes
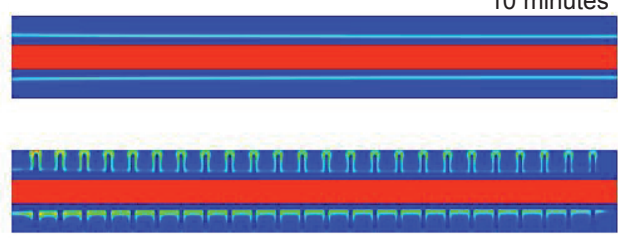

$\begin{array}{lllllllllll}0 & 0.1 & 0.2 & 0.3 & 0.4 & 0.5 & 0.6 & 0.7 & 0.8 & 0.9 & 1\end{array}$

Fig. 3. a) Temperature distribution $(\mathrm{K})$ and b) Liquid melting fraction within PCM thermal capacitor at time of 5 minutes and 10 minutes

Looking towards the temperature distributions within the PCM thermal capacitor during maximum and minimum inlet temperature, as presented in Fig. $3 \mathrm{a}$, it is found that PCM temperature is relatively constant around the melting temperature despite large changing in heat transfer fluid temperature. This relatively constant temperature is due to phase change process on the PCM side as presented in Fig. 3b. As observed, phase change occurs only in the area near the metal surface. This is expected as the PCM considered in this study, paraffin wax, has low thermal conductivity. Therefore heat transfer from the HTF to PCM occurs slowly. This finding implies that higher heat transfer in PCM side is required to achieve high performance PCM thermal capacitor.
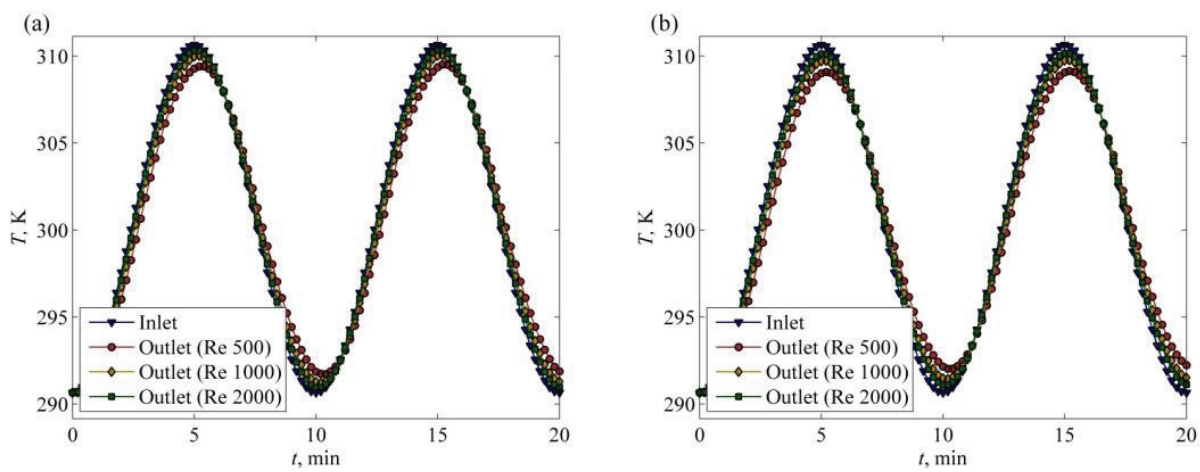

Fig. 4. Temperature fluctuation at inlet and outlet of PCM thermal capacitor a) with fin and b) without fin at various inlet Reynolds numbers

Next, the effect of inlet velocity on the performance of PCM thermal capacitor is evaluated. In this evaluation, three inlet velocities corresponding to inlet Reynolds number of 500, 1000 and 2000 is examined. Fig. 4 presents the temperature fluctuations at inlet and 
outlet for PCM thermal capacitor without and with fin at different inlet Reynolds numbers. As expected, higher Reynolds number yields in the lower temperature reduction. This is attributed to the fact that at lower velocity, the heat transfer fluid has longer heat transfer time.

\section{Conclusions}

A computational fluid dynamics (CFD) model which takes into account conjugate heat transfer between water as heat transfer fluid and phase change material as thermal capacitor is developed based on the validated model developed in previous study. Several thermal capacitor configurations were evaluated. Their performance is examined in term of reduction in temperature variation and induced pressure drop across the channel. The results indicate that PCM thermal capacitor without extended surface installed is capable to reduce temperature fluctuation by $\sim 1^{\circ} \mathrm{C}$. A larger reduction in temperature fluctuation is achieved when extended surface is installed in PCM. Subsequent study is currently underway to investigate various key factors affecting the performance of the proposed thermal capacitor. It should be noted that the mathematical model derived can easily be extended for other phase change material such as salt hydrate or metallic composite for high temperature application.

The authors gratefully acknowledge the facility and financial support from the Universiti Teknologi PETRONAS (UTP) through YUTP Fundamental Research Grant (YUTP-FRG) no 0153AA-E64.

\section{References}

1. A. Sharma, V. V. Tyagi, C. R. Chen and D. Buddhi. 2009. Renew Sustain Energy Rev 13(2), 318345 (2009).

2. B. Xu, P. Li, and C. Chan. 2015. Appl Energy 160, 286-307 (2015).

3. R. K. Sharma, P. Ganesan, V. V. Tyagi, H. S. C. Metselaar and S. C. Sandaran. Energy Convers Manage 95:193-228 (2015).

4. P. Zhang, X. Xiao and Z. W. Ma. 2016. Appl Energy 165, 472-510 (2016).

5. P. B. Salunke and P. S. Shembekar. 2012. Renew Sustain Energy Rev 16(8), 5603-5616 (2012).

6. F. Agyenim, N. Hewitt, P. Eames and M. A. Smyth. Renew Sustain Energy Rev 14(2), 615-628 (2010).

7. A. A. Al-Abidi, S. Mat, K. Sopian and M. Y. Sulaiman. 2013. Abdulrahman Th. Appl Therml Eng 53, 147-156 (2013).

8. G. Nardin, A. Meneghetti, F. Dal Magro and N. Benedetti. Appl Energy 136, 947-955 (2014).

9. F. Dal Magro, A. Meneghetti, G. Nardin and S. Savino. Energy Convers Manage 104, 78-89 (2015).

10. A. Novikov, D. Lexow and M. Nowottnick. 2014. "Cooling of electronic assemblies through PCM containing coatings". In: Proceedings of the $5^{\text {th }}$ Electronics SystemIntegration Technology Conference (Helsinki, Finland, 2014), pp. 1-5.

11. D. Verdiera, A. Ferrière, Q. Falcoz, F. Siros, and R. Couturier. Energy Procedia 49, 1044-1053 (2014).

12. J.C. Kurnia, A.P. Sasmito, S.V. Jangam, A.S. Mujumdar, Appl Therm Eng 50, 896-907 (2013). 
13. J.C. Kurnia, A. P. Sasmito, T. Shamim, A. S. Mujumdar. Appl Therm Eng 102,849-860 (2016).

14. J.C. Kurnia, A. P. Sasmito, A. S. Mujumdar. Heat Transfer Eng 37,1341-1356 (2016).

15. J.C. Kurnia, A. P. Sasmito, S. I. Ping. Energy Procedia 105, 4173-4178 (2017).

16. J.C. Kurnia, A. P. Sasmito. Appl Energy (doi: 10.1016/j.apenergy.2017.08.087) 